\title{
TITLE:
}

\section{On the S-matrix for three body Schrodinger Operators}

$\operatorname{AUTHOR}(\mathrm{S})$ :

ISOZAKI, Hiroshi

\section{CITATION:}

ISOZAKI, Hiroshi. On the S-matrix for three body Schrodinger Operators. 数理解析研究所講究録 1992, 779: 76-84

ISSUE DATE:

1992-04

URL:

http://hdl.handle.net/2433/82478

RIGHT: 


\title{
On the S-matrix for three body Schrödinger Operators
}

\author{
Hiroshi ISOZAKI \\ Department of Mathematics, Osaka University, Toyonaka, 560, \\ JAPAN
}

\subsection{Collision processes in the three-body problem}

We consider collision processes of quantum mechanical three particles labelled by $1,2,3$. Suppose in the initial state the two of them form a bound state, denoted by $(1,2)$, and the third particle collides with this pair. Then there occurs one of the following five phenomena:

$$
(1,2)+(3) \Longrightarrow \begin{cases}(a) & (1,2)+(3) \\ (b) & (1,2)^{*}+(3)_{*} \\ (c) & (1,2)^{\prime}+(3) \\ (d) & (1,3)+(2) \\ (e) & (1)+(2)+(3)\end{cases}
$$

(a) is an elastic process. In (b), the energy of the pair changes. In (c), the energy of the pair does not change, but this pair takes a different state (which happens when the eigenvalue is degenerate). (d) is a rearrangement process. Finally in (e), all of the three particles move freely after the collision. The first four cases are treated in essentially the same way as in the 2-body problem. So, we consider the properties of the S-matrix associated with the case (e).

\subsection{S-matrix}

In $\mathbf{R}^{3}$ we consider three particles with mass $m_{i}$ and position $x^{i}$. We choose a pair $(i, j)$ and denote it by $\alpha$. Let

$$
\frac{1}{m_{\alpha}}=\frac{1}{m_{i}}+\frac{1}{m_{j}}, \quad \frac{1}{n_{\alpha}}=\frac{1}{m_{k}}+\frac{1}{m_{i}+m_{j}}
$$

be the reduced masses and 


$$
x^{\alpha}=\sqrt{2 m_{\alpha}}\left(x^{i}-x^{j}\right), \quad x_{\alpha}=\sqrt{2 n_{\alpha}}\left(x^{k}-\frac{m_{i} x^{i}+m_{j} x^{j}}{m_{i}+m_{j}}\right)
$$

be the relative coordinates. Let

$$
X=\left\{\left(x^{1}, x^{2}, x^{3}\right) ; \sum_{i=1}^{3} m_{i} x^{i}=0\right\}
$$

Then in $L^{2}(X)$ the Schrödinger operator is given by

$$
H=H_{0}+\sum_{\alpha} V_{\alpha}\left(x^{\alpha}\right), \quad H_{0}=-\Delta_{x^{\alpha}}-\triangle_{x_{\alpha}} .
$$

If the pair potentials are short-range, the wave operators are known to exist:

$$
\begin{aligned}
& W_{0}^{ \pm}=\mathrm{s}-\lim _{t \rightarrow \pm \infty} e^{i t H} e^{-i t H_{0}}, \\
& W_{\alpha}^{ \pm}=\mathrm{s}-\lim _{t \rightarrow \pm \infty} e^{i t H} e^{-i t H_{\alpha}} J_{\alpha},
\end{aligned}
$$

where

$$
H_{\alpha}=H_{0}+V_{\alpha}, \quad\left(J_{\alpha} f\right)\left(x^{\alpha}, x_{\alpha}\right)=u_{\alpha}\left(x^{\alpha}\right) f\left(x_{\alpha}\right)
$$

$u_{\alpha}$ being a normalized eigenfunction of $h^{\alpha}=-\Delta_{x^{\alpha}}+V_{\alpha}\left(x^{\alpha}\right)$ with eigenvalue $E^{\alpha}<0$. The scattering operator $S_{0 \alpha}$ is defined by

$$
S_{0 \alpha}=\left(W_{0}^{+}\right)^{*} W_{\alpha}^{-} \text {. }
$$

To introduce the S-matrix, we use unitary operators $\mathcal{F}_{0}: L^{2}\left(\mathbf{R}^{6}\right) \rightarrow$ $L^{2}\left((0, \infty) ; L^{2}\left(S^{5}\right)\right)$ and $\mathcal{F}_{\alpha}: L^{2}\left(\mathbf{R}^{3}\right) \rightarrow L^{2}\left(\left(E^{\alpha}, \infty\right) ; L^{2}\left(S^{2}\right)\right)$ defined by

$$
\begin{gathered}
\left(\mathcal{F}_{0} f\right)(\lambda, \theta)=C_{0}(\lambda) \int_{\mathbf{R}^{8}} e^{-i \sqrt{\lambda} \theta \cdot x} f(x) d x \\
C_{0}(\lambda)=(2 \pi)^{-3} 2^{-1 / 2} \lambda \\
\left(\mathcal{F}_{\alpha} f\right)(\lambda, \omega)=C_{\alpha}(\lambda) \int_{\mathbf{R}^{3}} e^{-i \sqrt{\lambda-E^{\alpha}} \boldsymbol{\omega} \cdot x} f(x) d x \\
C_{\alpha}(\lambda)=(2 \pi)^{-3 / 2} 2^{-1 / 2}\left(\lambda-E^{\alpha}\right)^{1 / 4}
\end{gathered}
$$

Let

$$
\hat{S}_{0 \alpha}=\mathcal{F}_{0} S_{0 \alpha} \mathcal{F}_{\alpha}^{*}
$$

Then as is well-known, $\hat{S}_{0 \alpha}$ is decomposable, namely, for any $\lambda>0$, there exists a bounded operator $\hat{S}_{0 \alpha}(\lambda) \in \mathbf{B}\left(L^{2}\left(S^{2}\right) ; L^{2}\left(S^{5}\right)\right)$ such that

$$
\left(\hat{S}_{0 \alpha} f\right)(\lambda, \theta)=\left(\hat{S}_{0 \alpha}(\lambda) f(\lambda, \cdot)\right)(\theta)
$$

for a.e. $\lambda>0, \theta \in S^{5}$ and all $f \in L^{2}\left(\left(E^{\alpha}, \infty\right) ; L^{2}\left(S^{2}\right)\right)$. This $\hat{S}_{0 \alpha}(\lambda)$ is called the S-matrix. Note that this definition contains a sort of ambiguity. Two families of operators $\left\{\hat{S}_{0 \alpha}(\lambda)_{i}\right\}_{\lambda=0}^{\infty}(\mathrm{i}=1,2)$ define the same scattering 
operator $S_{0 \alpha}$, if $\hat{S}_{0 \alpha}(\lambda)_{1}=\hat{S}_{0 \alpha}(\lambda)_{2}$ for a.e. $\lambda>0$. The study of this family of operators $\left\{\hat{S}_{0 \alpha}(\lambda)\right\}_{\lambda=0}^{\infty}$ is not an easy problem. The general result known so far is that of Amrein-Pearson-Sinha [1] and Enss-Simon [5] asserting that $\hat{S}_{0 \alpha}(\lambda)$ is a Hilbert-Schmidt operator for a.e. $\lambda>0$, if the pair potentials decay faster than $\left|x^{\alpha}\right|^{-2}$. Our aim is to show that there is a representative $\left\{\hat{S}_{0 \alpha}(\lambda)\right\}_{\lambda=0}^{\infty}$ continuous in $\lambda>0$ and to investigate its detailed properties.

\subsection{Main results (1)}

We assume that $V_{\alpha}$ is a real $C^{\infty}$-function such that for a constant $\rho>0$

$$
\left|\partial_{y}^{m} V_{\alpha}(y)\right| \leq C_{m}(1+|y|)^{-\rho-m}, \quad m=0,1,2, \ldots,
$$

where $\partial_{y}^{m}$ denotes an arbitrary derivative of $m$-th order with respect to $y$, and $C_{m}$ is a constant. This assumption is stronger than actually needed. One can also allow certain local singularities for $V_{\alpha}$. Let

$$
X_{\beta}=\left\{x \in X ; x^{\beta}=0\right\},
$$

and define

$$
M=S^{5} \backslash \cup_{\beta} X_{\beta}, \quad N=S^{5} \cap\left(\cup_{\beta} X_{\beta}\right) .
$$

Theorem 1.1 (1) Suppose $\rho>4+1 / 2$. Then $\hat{S}_{0 \alpha}(\lambda)$ has a continuous kernel outside $N$ :

$$
\hat{S}_{0 \alpha}(\lambda ; \theta, \omega) \in C\left((0, \infty) \times M \times S^{2}\right) .
$$

(2) Suppose $\rho>5+1 / 2$. Let $\beta$ be any pair and decompose $\theta \in S^{5}$ as $\theta=\left(\theta^{\beta}, \theta_{\beta}\right)$ in accordance with the choice of the Jacobi-coordinates. Then as $\left|\theta^{\beta}\right| \rightarrow 0$,

$$
\hat{S}_{0 \alpha}(\lambda ; \theta, \omega) \simeq\left|\theta^{\beta}\right|^{-1} A_{\beta,-1}\left(\lambda ; \frac{\theta^{\beta}}{\left|\theta^{\beta}\right|}, \theta_{\beta}, \omega\right)+A_{\beta, 0}\left(\lambda ; \frac{\theta^{\beta}}{\left|\theta^{\beta}\right|}, \theta_{\beta}, \omega\right),
$$

where

$$
\begin{aligned}
& A_{\beta,-1}\left(\lambda ; \frac{\theta^{\beta}}{\left|\theta^{\beta}\right|}, \theta_{\beta}, \omega\right) \\
= & \sum_{j}^{\text {finite }} C_{\beta 1}^{(j)}\left(\lambda ; \theta_{\beta}, \omega\right) \times \int_{\mathbf{R}^{3}} \frac{\theta^{\beta}}{\left|\theta^{\beta}\right|} \cdot x^{\beta} V_{\beta}\left(x^{\beta}\right) u_{\beta}^{(j)}\left(x^{\beta}\right) d x^{\beta} \\
+ & C_{\beta 2}\left(\lambda ; \theta_{\beta}, \omega\right) \times \int_{\mathbf{R}^{3}} V_{\beta}\left(x^{\beta}\right) \varphi_{\beta}\left(x^{\beta}\right) d x^{\beta}
\end{aligned}
$$

$u_{\beta}^{(j)}$ being the eigenfunction with zero eigenvalue for $h^{\beta}$, and $\varphi_{\beta}$ the zeroresonance. $A_{\beta, 0}$ is continuous with respect to all of its arguments. $A_{\beta,-1}=$ 0 , if 0 is neither an eigenvalue nor the resonance for $h^{\beta}$. In this case, $\hat{S}_{0 \alpha}(\lambda ; \theta, \omega)$ is continuous at $\theta^{\beta}=0$. 
In the course of the proof, we shall see that $\hat{S}_{0 \alpha}(\lambda)$ is a $\mathbf{B}\left(L^{2}\left(S^{2}\right) ; L^{2}\left(S^{5}\right)\right)$ -valued continuous function of $\lambda>0$ if $\rho>5+1 / 2$. The zero-resonance $\varphi_{\beta}$ is the solution of the equation $h^{\beta} \varphi_{\beta}=0$ which behaves like $\varphi_{\beta} \simeq C /\left|x^{\beta}\right|$ as $\left|x^{\beta}\right| \rightarrow \infty, C \neq 0$. From our proof, one can see that if the pair potentials decay sufficiently rapidly, $\hat{S}_{0 \alpha}(\lambda ; \theta, \omega)$ is smooth on $M \times S^{2}$, but the zero eigenvalue and the zero-resonance are known to exist even if the potential is compactly supported. (See e.g. [3]). For the S-matrix from 2 to 3 cluster scattering, it is therefore the zero-eigenfunctions and the zero-resonances of subsystems that determines its singularities.

As for the coefficients $C_{\beta 1}^{(j)}\left(\lambda ; \theta_{\beta}, \omega\right)$ and $C_{\beta 2}\left(\lambda ; \theta_{\beta}, \omega\right)$, we have

Theorem 1.2 Up to a multiplicative constant depending only on $\lambda$ and $E^{\alpha}$, $C_{\beta 1}^{(j)}\left(\lambda ; \theta_{\beta}, \omega\right)$ and $C_{\beta 2}\left(\lambda ; \theta_{\beta}, \omega\right)$ coincide with the scattering amplitudes for 2 cluster scattering.

More precisely, $C_{\beta 1}^{(j)}\left(\lambda ; \theta_{\beta}, \omega\right)$ and $C_{\beta 2}\left(\lambda ; \theta_{\beta}, \omega\right)$ are the scattering amplitudes for 2-cluster scattering in which, after the collision, the pair $\beta$ becomes the bound state with zero energy or the zero-resonance, respectively. One should note that the notion of 2-cluster scattering associated with the zeroresonance is somewhat ambiguous since it has not yet been introduced in mathematical literatures. So, we shall begin by explaining the meaning of Theorem 1.2.

\subsection{Generalized eigenfunctions}

We first recall the 2-body problem. The generalized eigenfunction $\varphi(x, \xi)$ of $-\Delta+V$ in $\mathbf{R}^{n}$ is written as

$$
\begin{aligned}
\varphi(x, \xi) & =e^{i x \xi}-v, \\
v & =v(x, \xi)=\left(-\Delta+V-|\xi|^{2}-i 0\right)^{-1}\left(V(x) e^{i x \xi}\right) .
\end{aligned}
$$

The first term, $e^{i x \xi}$, represents the incident wave, and the second term, $v$, the scattered wave. The scattering amplitude $A(\lambda ; \theta, \omega)=\hat{S}(\lambda ; \theta, \omega)-\delta(\theta-\omega)$ is derived from $v$ in the following way :

$$
\begin{aligned}
v(x, \sqrt{\lambda} \omega) & \sim C(\lambda) r^{-(n-1) / 2} e^{i \sqrt{\lambda} r} A(\lambda ; \theta, \omega), \\
\theta & =x /|x|, \quad r=|x| \rightarrow \infty .
\end{aligned}
$$

In the case of the three body problem, our generalized eigenfunction is given by

$$
\begin{gathered}
\varphi(x, \lambda, \omega)=e^{i \sqrt{\lambda-E^{\alpha}} \omega \cdot x_{\alpha}} u_{\alpha}\left(x^{\alpha}\right)-v \\
v=R(\lambda+i 0) f, \quad R(z)=(H-z)^{-1} \\
f=f(x, \lambda, \omega)=\sum_{\gamma \neq \alpha} V_{\gamma}\left(x^{\gamma}\right) u_{\alpha}\left(x^{\alpha}\right) e^{i \sqrt{\lambda-E^{\alpha}} \omega \cdot x_{\alpha}} .
\end{gathered}
$$


We relate the coefficients $C_{\beta 1}^{(j)}, C_{\beta 2}$ appearing in Theorem 1.1 to the asymptotic properties of $v$.

For small positive constants $\epsilon$ and $\epsilon_{2}$, we take smooth functions $\chi_{\beta}(x)$ and $\psi_{\beta}(t)$ defined by

$$
\begin{gathered}
\chi_{\beta}(x)=\left\{\begin{array}{lll}
1 & \text { if } & \frac{\left|x^{\beta}\right|}{|x|}<2 \epsilon,|x|>1, \\
0 & \text { if } & \frac{\left|x^{\beta}\right|}{|x|}>3 \epsilon
\end{array}\right. \\
\psi_{\beta}(t)=\left\{\begin{array}{lll}
1 & \text { if } & |t-\lambda|<\epsilon_{2} \\
0 & \text { if } & |t-\lambda|>2 \epsilon_{2} .
\end{array}\right.
\end{gathered}
$$

We put

$$
w\left(x_{\beta}\right)=\int_{\mathbf{R}^{3}} u_{\beta}^{(j)}\left(x^{\beta}\right) \psi_{\beta}\left(D_{x_{\beta}}\right) \chi_{\beta}(x) v(x) d x^{\beta},
$$

where $\psi_{\beta}\left(D_{x_{\beta}}\right)$ is a pseudo-differential operator (Ps.D.Op. in short) with symbol $\psi_{\beta}\left(\left|\xi_{\beta}\right|^{2}\right)$, and $u_{\beta}^{(j)}\left(x^{\beta}\right)$ is an eigenfunction of $h^{\beta}$ with zero eigenvalue. Note that the integral is convergent since it is actually performed on the set $\left\{\left|x^{\beta}\right| \leq 2 \epsilon\left(1-4 \epsilon^{2}\right)^{-1}\left|x_{\beta}\right|\right\}$. Then we have

Lemma 1.3 The following asymptotic expansion holds :

$$
w\left(r \theta_{\beta}\right) \sim C(\lambda) r^{-1} e^{i \sqrt{\lambda} r} C_{\beta 1}^{(j)}\left(\lambda ; \theta_{\beta}, \omega\right),
$$

as $r=\left|x_{\beta}\right| \rightarrow \infty$ in $L^{2}\left(S^{2}\right)$. Moreover, up to a constant depending only on $\lambda, C_{\beta 1}^{(j)}\left(\lambda ; \theta_{\beta}, \omega\right)$ coincides with the scattering amplitude introduced by the time-dependent method.

One should note that it is not an obvious problem to construct the integral kernel of the 2-cluster scattering amplitude when the final state has the zero energy. The above lemma means that the kernel does exist and is equal to $C_{\beta 1}^{(j)}$.

Now, one can think of the collision process, in which, after the collision, the pair $\beta$ takes the zero-resonance state. It is not easy to define the associated scattering amplitude by the time-dependent method, since the zero-resonance, $\varphi_{\beta}$, does not belong to $L^{2}\left(\mathbf{R}^{3}\right)$. However, the stationary method explained above works equally well for this case. We define $\tilde{w}$ by

$$
\tilde{w}\left(x_{\beta}\right)=\int_{\mathbf{R}^{3}} \varphi_{\beta}\left(x^{\beta}\right) \psi_{\beta}\left(D_{x_{\beta}}\right) \chi_{\beta}(x) v(x) d x^{\beta} .
$$

By the same reasoning as above, this makes sense. We then have

Lemma 1.4 


$$
\tilde{w}\left(r \theta_{\beta}\right) \sim C(\lambda) r^{-1} e^{i \sqrt{\lambda} r} C_{\beta 2}\left(\lambda ; \theta_{\beta}, \omega\right),
$$

as $r=\left|x_{\beta}\right| \rightarrow \infty$ in $L^{2}\left(S^{2}\right)$.

Comparing the above two lemmas, it seems to be natural to call $C_{\beta 2}\left(\lambda ; \theta_{\beta}, \omega\right)$ the scattering amplitude associated with the zero-resonance, up to a constant factor. Actually, the above two lemmas are the content of Theorem 1.2.

\subsection{Main results (2)}

We continue the above point of view of studying the spatial asymptotic properties of $v$.

Theorem 1.5 If $\rho>4+1 / 2$, for any $\lambda>0$,

$$
\begin{aligned}
\mathrm{s}-\lim _{r \rightarrow \infty} r^{5 / 2} e^{-i \sqrt{\lambda} r} v(r \cdot) & =C(\lambda) \hat{S}_{0 \alpha}(\lambda ; \cdot, \omega), \\
C(\lambda) & =e^{\pi i / 4} 2 \pi \lambda^{-1 / 4}\left(\lambda-E^{\alpha}\right)^{-1 / 4},
\end{aligned}
$$

in $L_{l o c}^{2}(M)$.

So, the scattering matrix $\hat{S}_{0 \alpha}(\lambda)$ is obtained from the asymptotic behavior of $v$ in the same way as in the two body problem. However, it is not easy to replace $M$ by $S^{5}$ in the above theorem, since the behavior of $v$ in a neighborhood of $N$ is rather complicated. What we can expect is the limit in an averaged sense.

Let $\chi_{\beta}(x)$ be as in (1.13). We take $\rho_{+}(t) \in C^{\infty}\left(\mathbf{R}^{1}\right)$ such that $\rho_{+}(t)=1$ if $t>1-\epsilon, \rho_{+}(t)=0$ if $t<1-2 \epsilon$. We also take $\rho(t) \in C_{0}^{\infty}((0, \infty))$ such that $\rho(t)=1$ near $t=1$ and $\int_{0}^{\infty} \rho(t) d t=1$. In order to facilitate the proof, the pair potentials are assumed to be rapidly decreasing, but the following two theorems can of course be proved for more slowly decreasing potentials.

Theorem 1.6 Suppose that $V_{\alpha}$ 's are rapidly decreasing functions. Then

$$
\begin{aligned}
& \mathrm{s}-\lim _{R \rightarrow \infty} \frac{1}{R} \int_{\mathbf{R}^{6}} e^{-i \sqrt{\lambda} \theta \cdot x_{\beta}} \theta_{\beta} \cdot \hat{x}_{\beta} \rho_{+}\left(\frac{\theta_{\beta}}{\left|\theta_{\beta}\right|} \cdot \frac{x_{\beta}}{\left|x_{\beta}\right|}\right) \chi_{\beta}(x) \rho\left(\frac{\left|x_{\beta}\right|}{R}\right) v(x) d x \\
& =C(\lambda) \hat{S}_{0 \alpha}(\lambda ; \theta, \omega), \\
& C(\lambda)=-(4 \pi)^{-1} \lambda^{-1 / 2} C_{0}(\lambda)^{-1} C_{\alpha}(\lambda)^{-1},
\end{aligned}
$$

in $L^{2}\left(\tilde{N}_{\beta}\right)$, where $\tilde{N}_{\beta}$ is a small neighborhood of $N \cap X_{\beta}$ and $\hat{x}_{\beta}=x_{\beta} /\left|x_{\beta}\right|$.

In the neighborhood of the $X_{\beta}$-plane, there are two sorts of scattering, the 3-cluster scattering and the 2-cluster scattering. We can distinguish between them by changing the way of taking the limit at infinity of $v$. 
Let $u_{\beta l}\left(x^{\beta}\right)$ be a normalized eigenfunction of $h^{\beta}$ with eigenvalue $E_{l}^{\beta} \leq 0$. Let $A_{\beta l}\left(\lambda ; \theta_{\beta}, \omega\right)$ be the 2-cluster scattering amplitude associated with the process in which, after the colloision, the pair $\beta$ takes the bound state $u_{\beta l}$.

Theorem 1.7 Suppose that $V_{\alpha}$ 's are rapidly decreasing functions. For a small $\epsilon>0$, let $\psi_{\beta}(t) \in C^{\infty}\left(\mathbf{R}^{1}\right)$ be such that $\psi_{\beta}(t)=1$ if $t>\lambda-\epsilon, \psi_{\beta}(t)=0$ if $t<\lambda-2 \epsilon$. Let $\psi_{\beta}\left(D_{x_{\beta}}\right)$ be the Ps.D.Op. with symbol $\psi_{\beta}\left(\left|\xi_{\beta}\right|^{2}\right)$. Fix $a>0$ arbitrarily. Then as $r=\left|x_{\beta}\right| \rightarrow \infty$, we have the following asymptotic expansion:

$$
\begin{aligned}
\psi_{\beta}\left(D_{x_{\beta}}\right) v & \simeq \sum_{l} C_{\beta l}(\lambda) u_{\beta l}\left(x^{\beta}\right) r^{-1} e^{i \sqrt{\lambda-E_{l}^{\beta}}} A_{\beta l}\left(\lambda ; \theta_{\beta}, \omega\right), \quad \theta_{\beta}=x_{\beta} / r \\
C_{\beta l}(\lambda) & =2 \pi i\left(\lambda-E^{\alpha}\right)^{-1 / 4}\left(\lambda-E_{l}^{\beta}\right)^{-1 / 4}
\end{aligned}
$$

uniformly for $\left|x^{\beta}\right|<\alpha, \theta_{\beta} \in S^{2}$.

\subsection{Some remarks}

Amrein, Pearson and Sinha [1] showed that, for the N-body problem, the total cross-section with 2-cluster initial state is finite for almost all energy and derived its asymptotic properties in an averaged sense under the assumption that the potentials decay faster than $\left|x^{\alpha}\right|^{-2}$. See also Enss-Simon [5]. Amrein and Sinha [2] also showed that, for the three body problem, the total cross-section is finite for all $\lambda>0$ under the assumption that each 2-body subsystem has neither the zero eigenvalue nor the zero-resonance. Ito and Tamura [11] studied the semi-classical asymptotics for the total cross-section in distributional sense. All of these works treats the case of the initial state of 2-clusters, while Newton [18] and Yafaev [20] studied the structure of the S-matrix coressponding the collision process from 3-clusters to 3-clusters. Asymptotic properties of generalized eigenfunctions of threebody Schrödinger operators have been so far studied mainly by physicists. In the work of Newton [17], Theorems 1.5 and 1.7 were derived by intuitive arguments. If we consider the collision process of initial state of 3-clusters, we are led to consider the generalized eigenfunction formally defined by

$$
\psi(x, \lambda, \theta)=e^{i \sqrt{\lambda} \theta \cdot x}-R(\lambda+i 0) \sum_{\alpha} V_{\alpha} e^{i \sqrt{\lambda} \theta \cdot x} .
$$

The rigorous study of this generalized eigenfunction seems to be much harder. Nuttal [19] and Newton [17] gave precise explanations. One should also note the work of Mercuriev [16] of the three-body scattering theory for the Coulomb potential based on the stationary theory.

To prove the above theorems, following Isozaki-Kitada [10], we first localize the S-matrix in the phase space. An important role is played by the 
estimate of the resolvent of $H$ multiplied by Ps.D.Op's. For that purpose we utilize the estimate of Skibsted [15] on propagation properties in the phase space of $e^{-i t H}$, whose method is based on the work of Sigal-Soffer [14]. These estimates have been further refined by Gérard [6]. The singularities of $\hat{S}_{0 \alpha}(\lambda)$ arise from the low-energy asymptotics of 2-body subsystems studied by Jensen and Kato [12]. Another important tool is the spectral representation theory developed for 2-body Schrödinger operators, the key idea of which is to relate the generalized eigenfunction to the spatial symptotics of the resolvent of Schrödinger operators .

\section{References}

1 W.O.Amrein, D.B.Pearson and K.B.Sinha, Bounds on the total scattering cross-section for N-body systems, Nuovo Cimento 52 A (1979), 115-131.

2 W.O.Amrein and K.B.Sinha, On the three body scattering cross sections, J. Phy.A : Math. Gen. 15 (1982), 1567-1586.

3 C.L.Dolph, J.B.Macleod and D.Thoe, The analytic continuation of the resolvent kernel and scattering operator associated with the Schrödinger operator, J. Math. Anal. Appl., 16 (1966), 311-332.

4 V.Enss, Quantum scattering theory of two and three body systems with potentials of short and long range, Lecture Notes in Math. 1159, Springer, Berlin-Heidelberg-New York, (1985).

$5 \quad$ V.Enss and B.Simon, Finite total cross sections in non-relativistic quantum mechanics, Commun. Math. Phys. 76 (1980), 177-209.

6 C.Gérard, Sharp propagation estimates for N-particle systems, preprint (1991).

7 G.M.Graf, Asymptotic completeness for N-body short-range quantum systems : A new proof, Commun. Math. Phys. 132 (1990), 73-101.

8 H.Isozaki, Structures of S-matrices for three body Schrödinger operators, (to appear in Commun. Math. Phys.).

9 H.Isozaki, Asymptotic properties of generalized eigenfunctions for three body Schrödinger operators, preprint (1991).

10 H.Isozaki and H.Kitada, Scattering matrices for two-body Schrödinger operators, Scientific papers of the college of arts and sciences, Tokyo Univ. 35 (1985), 81-107.

11 H.T.Ito and H.Tamura, Semi-classical asymptotics for total scattering cross sections of 3-body systems, preprint (1990).

12 A.Jensen and T.Kato, Spectral properties of Schrödinger operators and time decay of the wave functions, Duke Math. J. 46 (1979), 583-611.

13 I.M. Sigal and A. Soffer, The N-particle scattering problem : Asymptotic completeness for short range quantum systems, Ann. of Math. 125 (1987), 35-108.

14 I.M.Sigal and A.Soffer, Local decay and propagation estimates for timedependent and time-independent Hamiltonians, preprint (1988).

15 E.Skibsted, Propagation estimates of N-body Schrödinger operators, Commun. Math. Phys. 142 (1991), 67-98. 
16 S.P.Mercuriev, On the three-body Coulomb scattering problem, Ann. Phys. 130 (1980), 395-426.

17 R.G.Newton, The asymptotic form of the three-particle wave functions and the cross sections, Ann. Phys. 74 (1972), 324-351.

18 R.G.Newton, The three particle S-matrix, J. Math. Phys. 15 (1974), 338343.

19 J.Nuttal, Asymptotic form of the three-particle scattering wave functions for free incident particles, J. Math. Phys. 12 (1971), 1896-1899.

20 D.Yafaev, On the multichannel scattering in two spàces, Theo. Math. Phys. 37 (1978), 867-874. 